\title{
Certified Clinical Nurse Specialist
}

National Cancer Institute

\section{Source}

National Cancer Institute. Certified Clinical Nurse Specialist. NCI Thesaurus. Code C71295.

A registered nurse who has a master's or doctorate degree in a clinical field of nursing and has been certified by the Board of Registered Nursing. 